\title{
CD73 promotes proliferation and migration of human cervical cancer cells independent of its enzyme activity
}

\author{
Zhao-wei Gao ${ }^{\dagger}$, Hui-ping Wang ${ }^{\dagger}$, Fang Lin, Xi Wang, Min Long, Hui-zhong Zhang ${ }^{*}$ and Ke Dong ${ }^{*}$
}

\begin{abstract}
Background: CD73 has both enzymatic and non-enzymatic functions in cells. As a nucleotidase, CD73 plays its enzymatic function by catalyzing the hydrolysis of AMP into adenosine and phosphate. In addition to this, accumulating data have shown that CD73 is a key regulatory molecule involved in cancer growth and metastasis, but this non-enzymatic function of CD73 in cervical cancer cells has not been well studied.

Methods: CD73 was overexpressed by pcDNA-NT5E expression vector transfection in Hela and SiHa cells. Cell's proliferation and migration were evaluated by MTT and scratch healing assay. The CD73 specific antagonist -APCP was used to inhibit CD73 enzymatic activity. And the effect of APCP on CD73 activity was determined by high performance liquid chromatography (HPLC). Expression level was assessed by qRT-PCR and western blotting.

Results: In the present study, we used Hela and SiHa cell lines to evaluate the effects of CD73 on cervical cancer cells proliferation and migration, and further explore the potential regulating mechanisms. Our data showed that CD73 overexpression significantly promoted cervical cancer cells proliferation and migration, and this promotive effect was not reverted by blocking CD73 enzymatic activity, both in Hela and SiHa cells. On the other hand, our data also showed that high concentration of adenosine inhibited Hela and SiHa cells proliferation and migration. These results demonstrated that the promotive effect of CD73 on cervical cancer cells proliferation and migration in vitro was independent from its enzymatic activity (i.e. production of adenosine). Furthermore, the expressions of EGFR, VEGF and Akt were significantly increased in CD73 overexpression Hela and SiHa cells.
\end{abstract}

Conclusions: Our data suggested that CD73 might promote proliferation and migration via potentiating EGFR/Akt and VEGF/Akt pathway, which was independent of CD73 enzyme activity. These data provide a novel insight into the regulating function of CD73 in cancer cells and suggest that CD73 may be promising therapeutic target in cervical cancer.

Keywords: CD73, Cervical cancer, Proliferation, Migration, Enzyme activity

\section{Background}

CD73 is a glycosylphosphatidylinositol (GPI) anchored cell surface protein, also known as ecto-5'-nucleotidase (ecto-5'-NT, EC 3.1.3.5). Accumulating data have shown that CD73 play important roles during cancer growth and metastasis [1-3]. CD73 has both enzymatic and non-enzymatic functions [4]. As an enzyme, CD73 catalyzes the AMP breakdown into adenosine. Notably, adenosine is an important purine signaling molecule

\footnotetext{
*Correspondence: huizz328@163.com; drdongke@163.com

${ }^{\dagger}$ Equal contributors

Department of Clinical Diagnosis, Tangdu Hospital, Fourth Military Medical University, Xinsi, Road, Xi'an, Shanxi 710038, China
}

which has been found to be involved in tumor immunoescape [5]. In addition to its enzymatic function, CD73 is also a regulatory molecule which related to cancer invasive and metastatic properties [3, 6, 7]. Studies have found that CD73 can promote proliferation and migration of several types of cancer cells $[6,8-10]$. Cervical cancer, a cancer arising from cervix is due to the abnormal proliferation of cells that have the ability to evade growth suppression. Cervical cancer is high in the rank of cancers affect women, with both the fourthhighest incidence and the fourth-highest fatality rate among women worldwide [11]. Infection with several special types of human papilloma virus (HPV) has been 
found to be the most high-risk cause of cervical cancer [12]. However, HPV infection was not enough to trigger cervical cancer. Other factors, like molecular alteration have also been found to play important role during cervical cancer development $[13,14]$. The role of CD73 in cervical cancer cells has not been well studied. In the present study, we investigated the effect of CD73 overexpression on cervical cancer cells proliferation and migration, and further explored its underlying regulatory mechanisms. Our data demonstrated that CD73 overexpression promoted Hela and $\mathrm{SiHa}$ cells proliferation and migration. Moreover, this promotive effect of CD73 should be via an enzymatic activity independent mechanism.

\section{Methods}

\section{Cell culture}

Two human cervical cancer cell lines, Hela and SiHa (American Type Culture Collection, ATCC), were used in this study. The cells were cultured in DMEM medium (Gibco, Carlsbad, NY, USA) supplemented with 10\% heat-inactivated Fetal bovine serum (FBS, Sijiqing Biotec, Hangzhou, China) at $37{ }^{\circ} \mathrm{C}$ with $5 \% \mathrm{CO}_{2}$ in a humidified incubator.

\section{pcDNA-NT5E recombinant expression vector construction and transfection}

CD73 coding gene - NT5E was cloned into the pcDNA3.0 + expression vector, then, the pcDNA-NT5E and control plasmids were transfected into Hela and $\mathrm{SiHa}$ cells using Lipofecta-mine $^{\text {rm }} 2000$ reagent (Invitrogen, Carlsbad CA, USA) following the protocol offered by the manufacturer. Briefly, parental Hela and SiHa cells $\left(3 \times 10^{5} /\right.$ well $)$ were plated in six - well plate and cultured until $70-80 \%$ confluence. Premixed lipofection and plasmid DNA $(10 \mu \mathrm{l}$ : $4 \mu \mathrm{g}$ ) were added into the wells and incubated for $24 \mathrm{~h}$. And then, the transfected cells were screened by G418.

\section{CD73 activity assay}

The conversion of AMP to adenosine was measured to assess the CD73 activity. Cultured cells were collected and suspended in DHank's buffer at a density of $4 \times 10^{5}$ cells/ ml. $500 \mu \mathrm{l}$ cell suspension was incubated with $1 \mathrm{mM}$ AMP at $37^{\circ} \mathrm{C}$ for $1 \mathrm{~h}$, the effect of APCP on CD73 activity was investigated by adding $50 \mu \mathrm{M}$ APCP into reaction system. Subsequently, cells were centrifuged, and the supernatants $(10 \mu \mathrm{l})$ were subjected to HPLC analysis (Shimadzu, LC-2030c; column: GL Sciences, C18, $3.5 \mu \mathrm{m}$, $2.5 \times 250 \mathrm{~mm}$ ). Adenosine was extracted through a $0-50 \%$ methanol $/ \mathrm{H}_{2} \mathrm{O}$ phase gradient elution $(1 \mathrm{ml} / \mathrm{min})$. The absorbance was measured at $259 \mathrm{~nm}$. The peak volume (PV) for adenosine was calculated using LabSolutions LC Workstation Ver.5.

\section{MTT cell viability assay}

The cells were seeded in 96 - well plate at a density of $4 \times$ $10^{3}$ cells per well and cultured at $37^{\circ} \mathrm{C}$ in DMEM medium plus 10\% FBS. At different time point, $10 \mu \mathrm{l}$ of MTT ( $5 \mathrm{mg} / \mathrm{ml}$, Amresco, Solon, USA) was added into each well and the cells were incubated for another $4 \mathrm{~h}$ at $37^{\circ} \mathrm{C}$. The supernatants were then discarded carefully and $150 \mu \mathrm{l}$ DMSO was added to each well. The plate was shaken for additional $10 \mathrm{~min}$ and the absorbance value was measured at $490 \mathrm{~nm}$ by using the microplate reader (Bio-Rad, Hercules, USA). The relative viability of cells was calculated as a percentage using the formula: (mean $\mathrm{OD}_{490}$ of treated cells/mean $\mathrm{OD}_{490}$ of control cells) $\times 100 \%$.

\section{Migration assays}

Migration ability of the cells was examined by scratch assay. Transfected and control cells were cultured in six-well plate. The scratch was performed with a pipette tip when cell density reached to $80 \%$. Once scratch was made, cells were gently washed by PBS twice, following cultured with serumfree medium. Images were captured immediately, and $24 \mathrm{~h}$ after the scratch was made. The cell migration distance was measured by HMIAS - 2000 software. The migration ability of cells was mirrored by relative migration ratio: Relative migration ratio $=($ Start distant - End distance $) /$ Start distance .

\section{Quantitative real-time RT-PCR and western-blot analysis}

Quantitative real-time RT-PCR was carried out using the SYBR Green kit (Invitrogen, Carlsbad, USA) according to the manufacturer's instructions. The relative expression levels of genes were normalized to the endogenous housekeeping gene $\beta$-actin. The primer sequence are list in Table 1. For western-blot, the total cell protein was loaded for SDS-PAGE, and then transferred onto polyvinylidene difluoride membranes. Membranes were blocked with 5\% non-fat dried milk for $2 \mathrm{~h}$ at room temperature, and then incubated with primary antibody ( $\mathrm{R} \& \mathrm{D}$ Systems, Inc.) overnight at $4{ }^{\circ} \mathrm{C}$. After washed with TBST for three times, membranes were incubated with secondary antibody for $2 \mathrm{~h}$ at room temperature. After washed with TBST, proteins were detected with western blotting luminol reagent (Santa Cruz Biotechnology, Santa Cruz, USA), $\beta$-actin were used as the internal standard.

\begin{tabular}{lll}
\multicolumn{3}{l}{ Table $\mathbf{1}$ primer sequence used in the study } \\
\hline Gene name & Forward primer (5'-3') & Reverse primer (5'-3') \\
\hline CD73 & GCCTGGGAGCTACGATTTG & TAGTGCCCTGGTACTGGTCG \\
EGFR & CCAAGGCACGAGTAACAA & ACATAACCAGCCACCTCC \\
VEGF & TTGCCTTGCTGCTCTACCTC & TGCATGGTGATGTTGGACTC \\
Akt1 & ACTGTCATCGAACGCACCTT & TTCTGCAGGACGCGGTCTC \\
$\beta$-actin & TGACGTGGACATCCGCAAAG & CTGGAGGTGACAGCGAGG \\
\hline
\end{tabular}



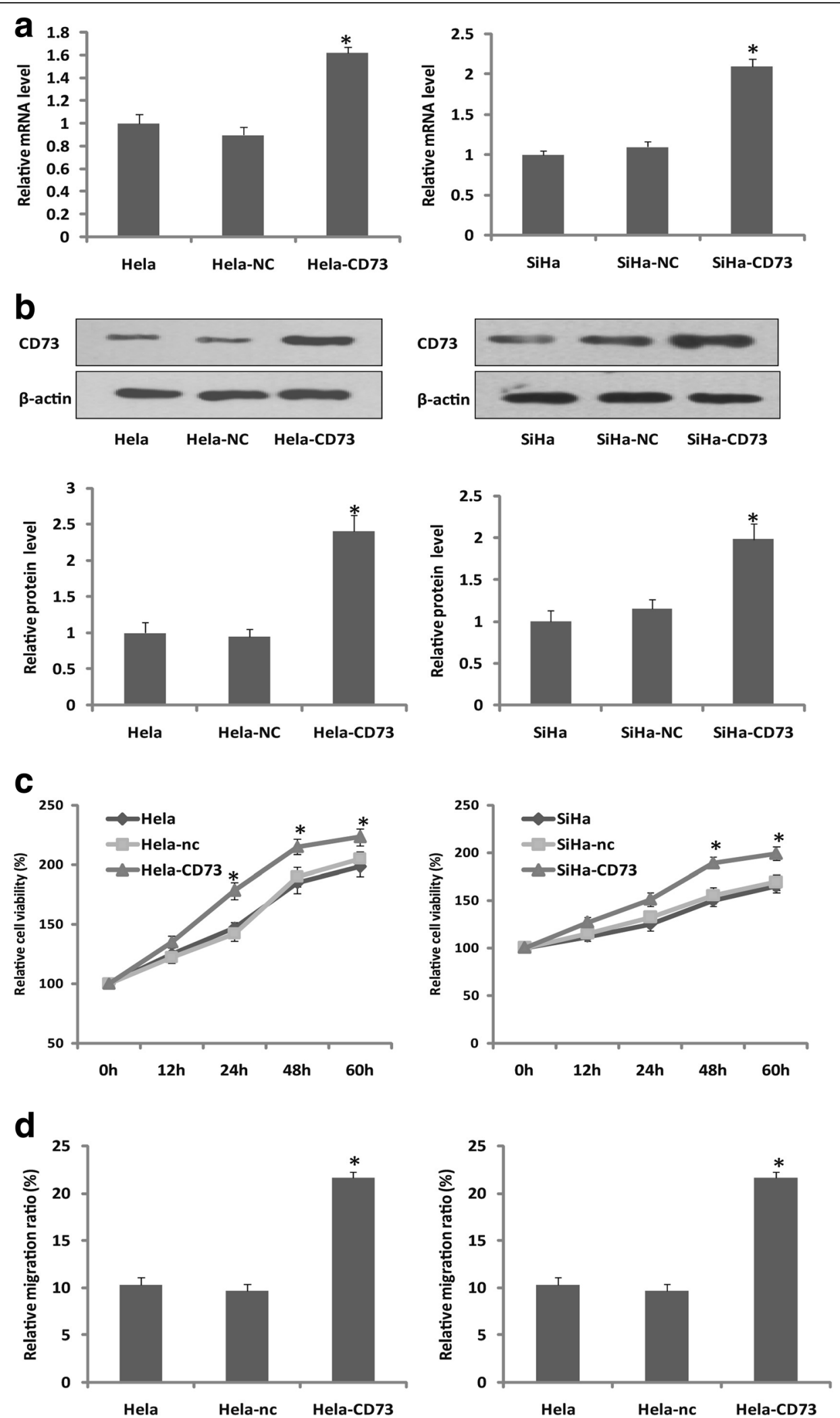

Fig. 1 The proliferation and migration of CD73 overexpression cells. a, b RT-PCR and western-blot showed that CD73 expression in transfected cells (named Hela-CD73 and SiHa-CD73) was significantly high than that in control cells. c MTT assay showed that CD73 overexpression promoted Hela and SiHa cells proliferation. $\mathbf{d}$ Scratch healing assay showed that CD73 overexpression promoted Hela and SiHa cells migration 


\section{Statistical analysis}

All data were confirmed in three biological replicates. The data are expressed as the mean \pm standard error. Data comparisons were conducted by using the Student's $t$-test. $P<0.05$ was considered to be statistically significant.

\section{Results}

\section{CD73 overexpression promoted proliferation and} migration of cervical cancer cells

To determine the effect of CD73 in proliferation and migration of human cervical cancer cells in vitro, the pcDNA-NT5E and control vectors were constructed and transfected into Hela and $\mathrm{SiHa}$ cells. The expression level of CD73 in transfected cells was significantly higher than that in mock and parental control cells (Fig. 1a), and the CD73 enzymatic activity was also increased in CD73 overexpressed cancer cells (Additional file 1: Figure S1). Then, the effect of CD73 overexpression on cervical cancer cells proliferation and migration was examined. By using MTT assay, we found that the proliferation rate of CD73 overexpression cells was significantly higher than that of control cells (Fig. 1b). Moreover, in addition to the promotive effect of CD73 on Hela and
SiHa cells proliferation, we also found that CD73 overexpression enhanced the Hela and SiHa cells migration ability by using scratch healing assay (Fig. 1c). These results demonstrated that CD73 overexpression promoted cervical cancer cells proliferation and migration in vitro.

\section{Blocking of CD73 enzyme activity could not revert its promotive effect on cells proliferation and migration} CD73 has both enzymatic and non-enzymatic function. To further investigate whether the promotive effect of CD73 on the cells proliferation and migration was dependent on its enzyme activity or was mainly the results of its non-enzymatic function, we used $50 \mu \mathrm{M}$ APCP (the specific inhibitor of CD73 enzymatic activity) to block the CD73 enzyme activity in the present study. HPLC analysis showed that APCP could inhibit CD73 enzymatic activity significantly. (Additional file 1: Figure S1). Moreover, APCP treatment did not influence the proliferation and migration of CD73 transfected Hela and SiHa cells (Fig. 2), which means that the promotive effect of CD73 is not reverted by blocking of CD73 enzymatic activity. These results indicated that the promotive effect of CD73 overexpression on the proliferation and migration of Hela and $\mathrm{SiHa}$ cells was independent of its enzyme activity.
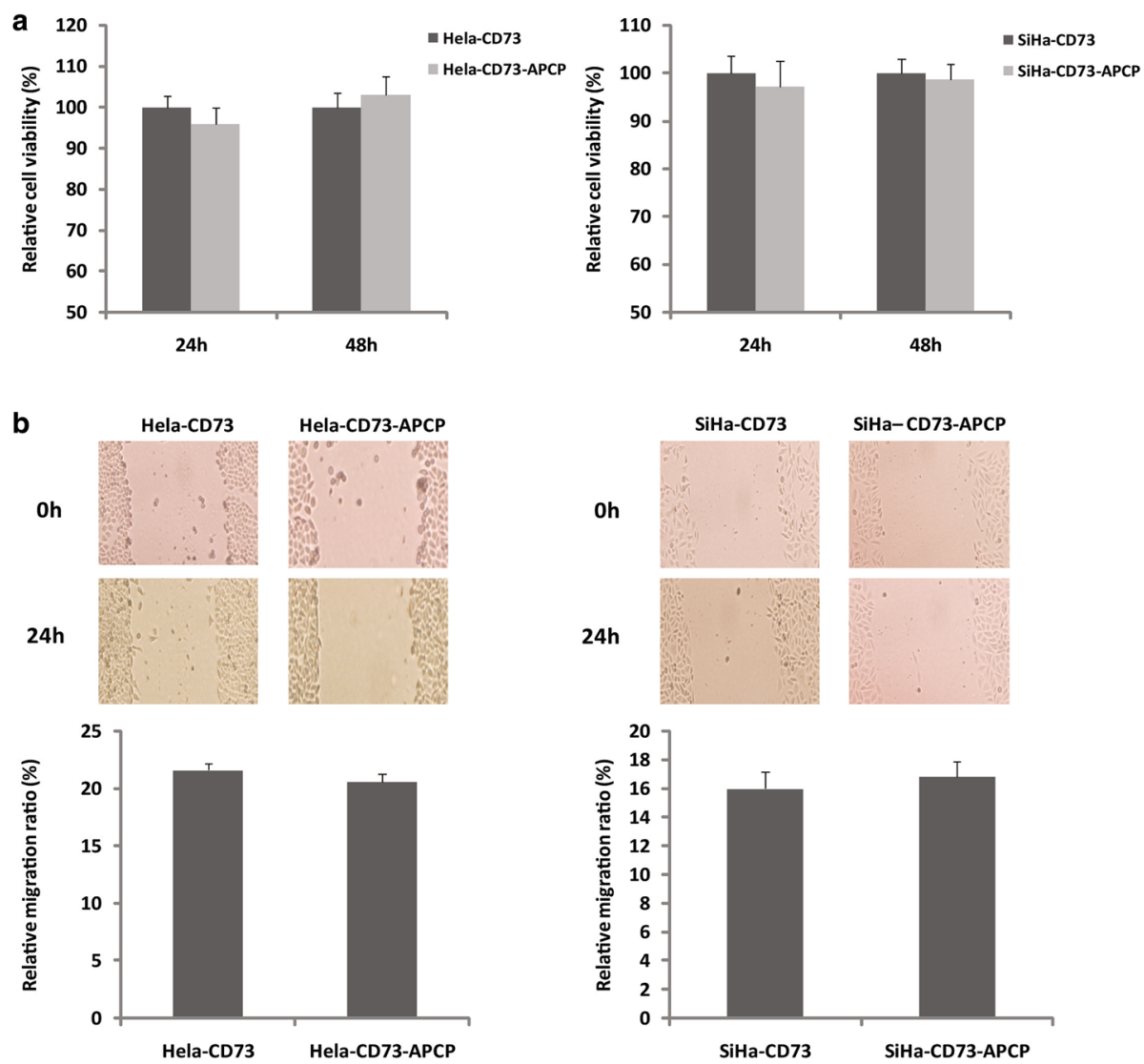

Fig. 2 The effect of APCP on CD73 transfected Hela and SiHa cells. a MTT assay showed that APCP treatment did not influence the proliferation of CD73 overexpression Hela and SiHa cells. $\mathbf{b}$ The migration of CD73 transfected cells was not changed in response to APCP treatment. These results suggested that APCP treatment did not revert the promotive effect of CD73 overexpression 
High concentration of extracellular adenosine decreased cervical cancer cells proliferation and migration

As a nucleotidase, CD73 catalyzed AMP breakdown into adenosine. In present study, we examined the effect of adenosine in cervical cancer cells proliferation and migration in vitro. Hela and $\mathrm{SiHa}$ cells were treated with different concentrations (1, 10, 50, $100 \mathrm{nM} ; 1,5,10,100,500 \mu \mathrm{M}$; $1,5,10 \mathrm{mM}$ ) of adenosine for $24 \mathrm{~h}$ and $48 \mathrm{~h}$. After adenosine treatment, MTT assay was used to detect the cancer cells proliferation. As shown in Fig. 3, compared with control cells, the proliferation rate was inhibited by high concentration $(>100 \mu \mathrm{M})$ of extracellular adenosine in both Hela and SiHa cells, while the cells proliferation was not influenced by low concentration $(<100 \mu \mathrm{M})$ of adenosine. Moreover, treatment with high concentration of adenosine significantly diminished migration ability of Hela and SiHa cells (Fig. 3). These results suggested that the promotive effect of $\mathrm{CD} 73$ overexpression on proliferation and migration in vitro was independent of adenosine (i.e. CD73 enzyme activity).

\section{CD73 overexpression potentiated EGFR/Akt and VEGF/Akt pathway}

Since the promotive effect of CD73 on the cells proliferation and migration was independent of its enzyme activity, there should be other mechanism involved in this promotive function. EGFR and VEGF are crucial signaling molecules which have been believed to promote cancer cell growth and metastasis. In this present study, we examined the effect of CD73 overexpression on EGFR and VEGF expression in cervical cancer cells. As shown in Fig. 4, compared with control cells, the EGFR expression was increased by $62 \%$ and $45 \%$, while VEGF was increased by $76 \%$ and 69\% in CD73 transfected Hela and SiHa cells. As Akt is an important downstream signaling molecule of EGFR and VEGF, we also investigated the change of Akt expression in CD73 overexpression cervical cancer cells. The RT-PCR and western-blot results showed that the expression of both Akt and phospho-Akt were significantly increased in CD73 transfected Hela and SiHa cells than that in control cells. These data suggested that CD73 overexpression potentiated EGFR/Akt and VEGF/Akt pathway. Furthermore, to rule out the possibility that these signaling molecules changes were induced by the CD73 enzyme activity, we examined the effect of APCP treatment on EGFR, VEGF and Akt expression. As shown in Fig. 4, the mRNA and protien expressions of these molecules in CD73 overexpression cells were not changed in response to APCP treatment (Fig. 4a, b). And moreover, we also investigated whether adenosine changed these molecules levels by qRT-PCR, the results were negative
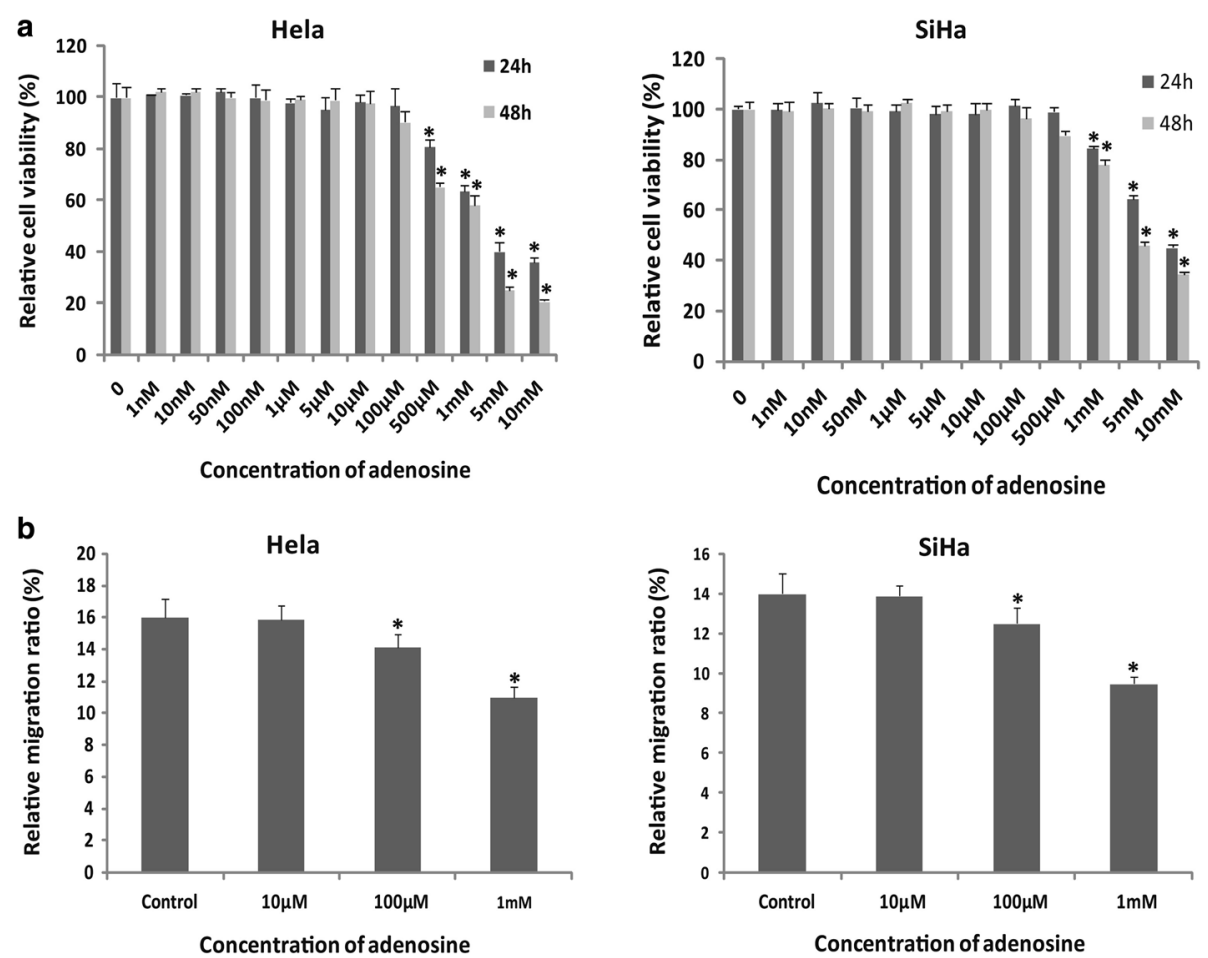

Fig. 3 The effect of extracellular adenosine on cells proliferation and migration. a Gradient adenosine treated Hela and SiHa cells. The results showed that high concentration of adenosine inhibits cells proliferation. $\mathbf{b}$ Scratch healing assay showed that high concentration of adenosine diminished the migration of Hela and SiHa cells 

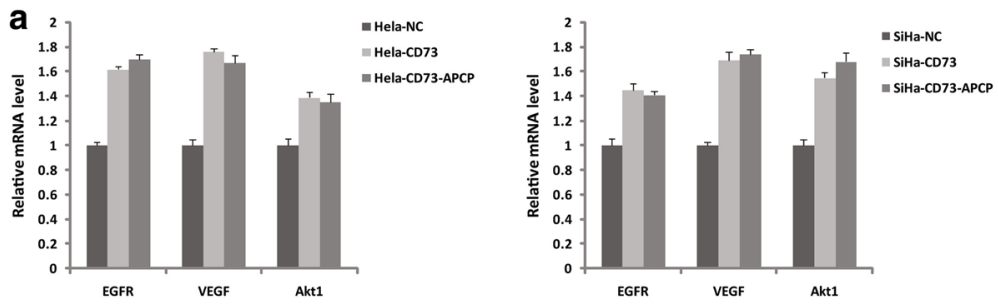

b
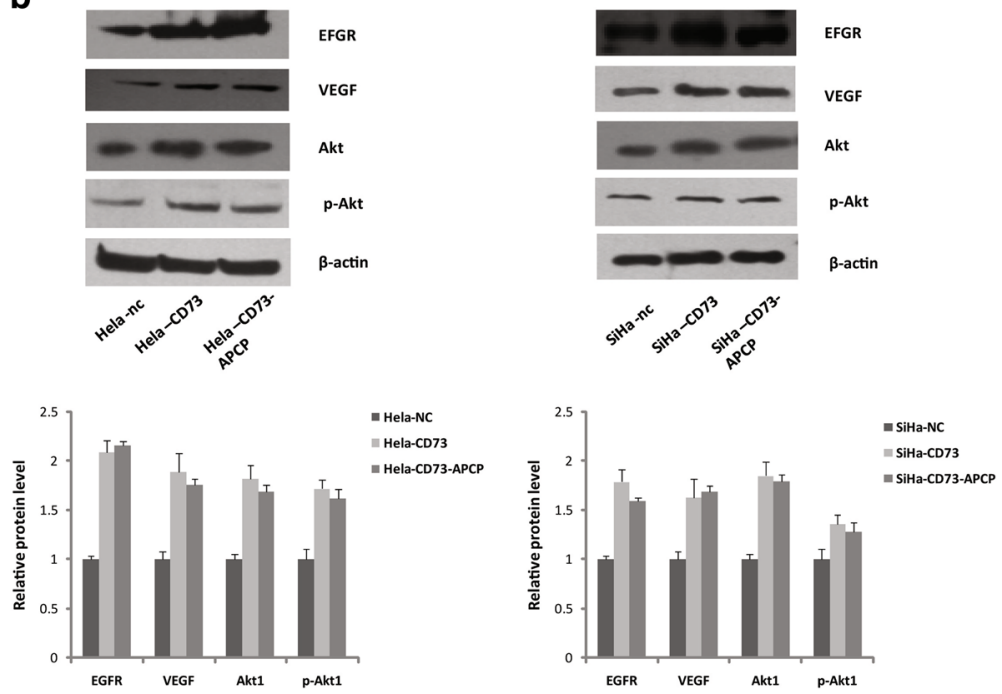

Fig. 4 CD73 overexpression potentiated EGFR/Akt and VEGF/Akt pathway. a RT-PCR and $\mathbf{b}$ western-blot showed that the expressions of EGFR, VEGF, Akt, pAkt were significantly higher in CD73 overexpression Hela and SiHa cells in compared with control cells, and moreover, increased effect in CD73 overexpression cells was not reverted by APCP treatment

(Additional file 2: Figure S2). Thus taken together, these data indicated that CD73 ovexpression promoted Hela and $\mathrm{SiHa}$ cells proliferation, at least partially, via potentiating the EGFR/Akt and VEGF/Akt signaling pathway, independent of CD73 enzymatic activity.

\section{Discussion}

CD73 has emerged as an important molecule during cancer growth and metastasis. This study was undertaken to evaluate the effect of CD73 on cervical cancer cells proliferation and migration. Our results showed that CD73 overexpression promoted cervical cancer cells proliferation and migration in vitro. CD73 has both enzymatic and non-enzymatic functions in cells. As a nucleotidase, CD73 catalyzes AMP breakdown into adenosine. CD73-generated adenosine has been recognized an important purinergic signaling during cancer progression, which can suppress immune response and assist tumor immune evasion in vivo via multiple pathway [5]. However, besides evading immune destruction, rapid and un-controlled proliferation of tumor cells is another key factor for cancer growth [15]. Regard to this, recent years, researchers investigated the direct effect of extracellular adenosine on cancer cells proliferation in vitro by adding adenosine into culture medium, these data, including ours here, have shown that high concentration of adenosine can inhibit tumor cells proliferation and migration [16-19]. However, this inhibiting effect of high concentration of adenosine is unexpected, since CD73 overexpression has been found to promote tumor cells proliferation and migration in vitro.

At first sight, the promotive effect of CD73 and inhibiting effect of adenosine on cervical cancer cells proliferation and migration seemed to be paradoxical. This apparent discrepancy could be explained on the basis of the following considerations. The concentration of adenosine is determined by a complex ectoenzyme mechanism. The generation and degradation of adenosine is catalyzed by a cascade of enzyme as follows: ATP/ADP breakdown into AMP by CD39, AMP breakdown into adenosine by CD73, and adenosine breakdown into inosine by ADA and its cofactor CD26. Thus, the increased concentration of adenosine by CD73 overexpression may be not enough to inhibit the cancer cells proliferation and migration (the adenosine concentration should be $>100 \mu \mathrm{M}$, which can display inhibited effects). As the low concentration of adenosine has no effect on cancer cells proliferation and migration, therefore, the promotive effect of CD73 overexpression on cells proliferation and 
migration in vitro were independent of its enzyme activity. Indeed, here we proved this hypothesis in Hela and $\mathrm{SiHa}$ cells. In present study, we found that the promotive effect of CD73 overexpression on cervical cancer cells proliferation and migration were not reverted by blocking of CD73 enzyme activity. These data suggested that CD73 promoted cervical cancer cells proliferation and migration, which was dependent of its non-enzyme activity. EGFR and VEGF have been believed to promote cancer growth and metastasis [20-23]. Co-expression of CD73 and EGFR or VEGF has been found in other type of cancers [24-26]. Consistently, in this study, we found that EGFR and VEGF expression were markedly increased in CD73 transfected cervical cancer cells. Although Zhi X's data have suggested that CD73 may promote EGFR expression though regulating some associated transcription factor, such as PPAR $\gamma$ [24]. However, the relevant studies are still few to demonstrate the regulated mechanism of CD73 on expression of EGFR and VEGF. The interaction among them needs further studies. Moreover, the Akt expression, which is an important downstream molecule of EGFR and VEGF, was also significantly higher in CD73 overexpression cells than that in control cells [27]. In the mean while, the EGFR, VEGF and Akt expression in CD73 overexpression cells were not changed in response to APCP treatment. In summary, our data suggest that CD73 overexpression promote cervical cancer cells proliferation and migration, via potentiating EGFR/Akt and VEGF/Akt pathway, independent of its enzymatic activity. These data provide a novel insight into the regulating function of CD73 in cancer and suggest that CD73 may be promising therapeutic target in cervical cancer.

\section{Conclusions}

Our data showed that CD73 promoted cervical cells proliferation and migration, and this promotive effect was independent of CD73 enzyme activity. In addition, we also showed that CD73 overexpression potentiated EGFR/Akt and VEGF/Akt pathway. These data provide a novel insight into the regulating function of CD73 in cancer cells and suggest that CD73 may be promising therapeutic target in cervical cancer.

\section{Additional files}

Additional file 1: Figure S1. CD73 enzymatic activity was assessed by using HPLC analysis. The catalyzed activity was increased in CD73 overexpressed Hela and SiHa cells, while was inhibited by APCP treatment. (JPG $1761 \mathrm{~kb}$ )

Additional file 2: Figure S2. $100 \mu \mathrm{M}$ Adenosine treatment did not change the expression of EGFR, VEGF and Akt. (JPG $335 \mathrm{~kb}$ )

\section{Abbreviations}

ATCC: American Type Culture Collection; FBS: Fetal bovine serum; HPLC: High performance liquid chromatography; HPV: Human papilloma virus; PV: Peak volume; qRT-PCR: Quantitative real-time polymerase chain reaction

\section{Acknowledgments}

This work is supported by the National Natural Science Fund of China (No. 81172148).

\section{Availability of data and materials}

All data generated or analysed during this study are included in this published article [and its Additional files 1 and 2].

\section{Authors' contributions}

ZWG and HP. Wang designed the experiments with help from KD. HPW, FL, $\mathrm{ML}$ and XW performed the experiments. ZWG interpreted data and wrote the manuscript with help from HZZ and KD. All authors read and approved the final version of this manuscript.

\section{Competing Interests}

The authors declare that they have no conflict of interest.

\section{Consent for publication}

Not applicable.

Ethics approval and consent to participate

Not applicable.

Received: 16 January 2016 Accepted: 8 February 2017 Published online: 15 February 2017

\section{References}

1. Gao ZW, Dong K, Zhang HZ. The roles of CD73 in cancer. Biomed Res Int. 2014;2014:460654.

2. Antonioli L, Hasko G, Fornai M, Colucci R, Blandizzi C. Adenosine pathway and cancer. where do we go from here? Expert Opin Ther Targets. 2014; 18(9):973-7.

3. Zhang B. CD73 promotes tumor growth and metastasis. Oncoimmunology. 2012;1(1):67-70.

4. Sadej R, Skladanowski AC. Dual, enzymatic and non-enzymatic, function of ecto-5'-nucleotidase (eN, CD73) in migration and invasion of A375 melanoma cells. Acta Biochim Pol. 2012;59(4):647-52.

5. Antonioli L, Blandizzi C, Pacher P, Hasko G. Immunity, inflammation and cancer: a leading role for adenosine. Nat Rev Cancer. 2013;13(12):842-57.

6. Wang L, Zhou X, Zhou T, Ma D, Chen S, Zhi X, Yin L, Shao Z, Ou Z, Zhou P. Ecto-5'-nucleotidase promotes invasion, migration and adhesion of human breast cancer cells. J Cancer Res Clin Oncol. 2008;134(3):365-72.

7. Terp MG, Olesen KA, Arnspang EC, Lund RR, Lagerholm BC, Ditzel HJ, LethLarsen R. Anti-human CD73 monoclonal antibody inhibits metastasis formation in human breast cancer by inducing clustering and internalization of CD73 expressed on the surface of cancer cells. J Immunol. 2013;191(8):4165-73.

8. Bavaresco L, Bernardi A, Braganhol E, Cappellari AR, Rockenbach L, Farias PF, Wink MR, Delgado-Canedo A, Battastini AM. The role of ecto-5'nucleotidase/CD73 in glioma cell line proliferation. Mol Cell Biochem. 2008; 319(1-2):61-8.

9. Zhou P, Zhi X, Zhou T, Chen S, Li X, Wang L, Yin L, Shao Z, Ou Z. Overexpression of Ecto-5'-nucleotidase (CD73) promotes T-47D human breast cancer cells invasion and adhesion to extracellular matrix. Cancer Biol Ther. 2007;6(3):426-31.

10. Zhi X, Chen S, Zhou P, Shao Z, Wang L, Ou Z, Yin L. RNA interference of ecto-5'-nucleotidase (CD73) inhibits human breast cancer cell growth and invasion. Clin Exp Metastasis. 2007;24(6):439-48.

11. Mello Pde A, Filippi-Chiela EC, Nascimento J, Beckenkamp A, Santana DB, Kipper F, Casali EA, Nejar Bruno A, Paccez JD, Zerbini LF, et al. Adenosine uptake is the major effector of extracellular ATP toxicity in human cervical cancer cells. Mol Bio Cell.2014;25(19):2905-18.

12. Rosa MI, Medeiros LR, Rosa DD, Bozzeti MC, Silva FR, Silva BR. Human papillomavirus and cervical neoplasia. Cad Saude Publica. 2009;25(5):953-64

13. Luhn P, Walker J, Schiffman M, Zuna RE, Dunn ST, Gold MA, Smith K, Mathews C, Allen RA, Zhang $R$, et al. The role of co-factors in the progression from human papillomavirus infection to cervical cancer. Gynecol Oncol. 2013;128(2):265-70.

14. Deng B, Zhang S, Miao Y, Zhang Y, Wen F, Guo K. Down-regulation of Frizzled-7 expression inhibits migration, invasion, and epithelial- 
mesenchymal transition of cervical cancer cell lines. Med Oncol (Northwood, London, England). 2015;32(4):102.

15. Hanahan D, Weinberg RA. Hallmarks of cancer: the next generation. Cell. 2011;144(5):646-74.

16. Yang D, Song J, Wu L, Ma Y, Song C, Dovat S, Nishizaki T, Liu J. Induction of senescence by adenosine suppressing the growth of lung cancer cells. Biochem Biophys Res Commun. 2013;440(1):62-7.

17. Shirali S, Aghaei M, Shabani M, Fathi M, Sohrabi M, Moeinifard M. Adenosine induces cell cycle arrest and apoptosis via cyclinD1/Cdk4 and Bcl-2/Bax pathways in human ovarian cancer cell line OVCAR-3. Tumour Biol. 2013; 34(2):1085-95.

18. Aghaei M, Karami-Tehrani F, Panjehpour M, Salami S, Fallahian F. Adenosine induces cell-cycle arrest and apoptosis in androgen-dependent and -independent prostate cancer cell lines, LNcap-FGC-10, DU-145, and PC3. Prostate. 2012:72(4):361-75.

19. Virtanen SS, Kukkonen-Macchi A, Vainio M, Elima K, Harkonen PL, Jalkanen S, Yegutkin GG. Adenosine inhibits tumor cell invasion via receptorindependent mechanisms. Mol Cancer Res. 2014;12(12):1863-74.

20. Zahonero C, Sanchez-Gomez P. EGFR-dependent mechanisms in glioblastoma: towards a better therapeutic strategy. Cell Mol Life Sci. 2014; 71(18):3465-88.

21. Yang R, Wu Y, Wang M, Sun Z, Zou J, Zhang Y, Cui H. HDAC9 promotes glioblastoma growth via TAZ-mediated EGFR pathway activation. Oncotarget. 2015;6(10):7644-56.

22. Qi L, Xing LN, Wei X, Song SG. Effects of VEGF suppression by small hairpin RNA interference combined with radiotherapy on the growth of cervical cancer. Genet Mol Res. 2014;13(3):5094-106.

23. Wang Q, Wang S, Sun SQ, Cheng ZH, Zhang Y, Chen G, Gu M, Yao HJ, Wang Z, Zhou J, et al. The effects of RNA interference mediated VEGF gene silencing on biological behavior of renal cell carcinoma and transplanted renal tumor in nude mice. Cancer Biomark : section A of Dis markers. 2016;16(1):1-9.

24. Zhi X, Wang Y, Yu J, Yu J, Zhang L, Yin L, Zhou P. Potential prognostic biomarker $\mathrm{CD} 73$ regulates epidermal growth factor receptor expression in human breast cancer. IUBMB Life. 2012;64(11):911-20.

25. Wu R, Chen Y, Li F, Li W, Zhou H, Yang Y, Pei Z. Effects of CD73 on human colorectal cancer cell growth in vivo and in vitro. Oncol Rep. 2016;35(3):1750-6.

26. Koszalka P, Golunska M, Stanislawowski M, Urban A, Stasilojc G, Majewski M, Wierzbicki P, Skladanowski AC, Bigda J. CD73 on B16F10 melanoma cells in CD73-deficient mice promotes tumor growth, angiogenesis, neovascularization, macrophage infiltration and metastasis. Int J Biochem Cell Biol. 2015;69:1-10.

27. Ohnishi Y, Yasui H, Kakudo K, Nozaki M. Cetuximab-resistant oral squamous cell carcinoma cells become sensitive in anchorage-independent culture conditions through the activation of the EGFR/AKT pathway. Int I Oncol. 2015;47(6):2165-72.

\section{Submit your next manuscript to BioMed Central and we will help you at every step:}

- We accept pre-submission inquiries

- Our selector tool helps you to find the most relevant journal

- We provide round the clock customer support

- Convenient online submission

- Thorough peer review

- Inclusion in PubMed and all major indexing services

- Maximum visibility for your research

Submit your manuscript at www.biomedcentral.com/submit

) Biomed Central 